\section{Hutchinson-Gilford Progeria Syndrome with Bilateral Blephroptosis and Cataracts}

Sir,

Progeria is a rare, autosomal dominant, deadly childhood disorder. It is one of the progeroid syndromes also known as Hutchinson-Gilford Progeria Syndrome (HGPS). Ageing is a developmental process that begins with fertilisation and ends up with death, involving a lot of environmental and genetic factors. ${ }^{1} \mathrm{It}$ is not necessarily a single gene disorder; there are multiple genes as well as there are cases with partial penetrance. ${ }^{2}$

According to literature, patients with progeria syndrome usually die in early twenties and never develop cataracts. ${ }^{3}$

A 40-year lady admitted in medical unit was diagnosed as a case of progeria syndrome. The diagnosis was made after detailed history and examination by the medical, gynecology, pediatrics, radiology, dermatology and pathology departments. So, the diagnosis was made after consultation with all relevant disciplines. The clinical features included short stature, micrognathia, dry course skin, facial features resembling an old person, alopecia, parrot beak nose, hearing difficulty and hyperlipidemia. The genetic testing was advised but after few days, the patient expired due to sudden cardiac failure.

She was referred to ophthalmology unit for her ocular problems. On examination; she had dimness of vision. Vision in both eyes was hand motions. On slit lamp examination, anterior chambers were shallow. Ocular adnexae and ocular motility were normal. There was bilateral moderate ptosis. Cornea and conjunctiva were normal. Pupils were round and reactive. Intraocular pressure was not recorded because of problems with communication. There were dense white cataracts in both eyes (Figure 1). B scan ultrasound was normal. She was advised cataract surgery. As she was deaf, thus it was impossible to be operated under local anesthesia. But due to high risk of anesthesia complications, relatives of the patient were not willing for surgery under general anesthesia. She was discharged on the will of the patient without surgery. Unfortunately, she died few months later.

Common clinical characteristics of progeria syndrome include extremely short stature, decreased body weight, alopecia, lipodystrophy, scleroderma, impaired joint mobility and bone lysis. Facial features resemble an aged person. Such patients die in the early twenties because of these complications. Survival for more than 20 years is rare and only a single case of survival for 45

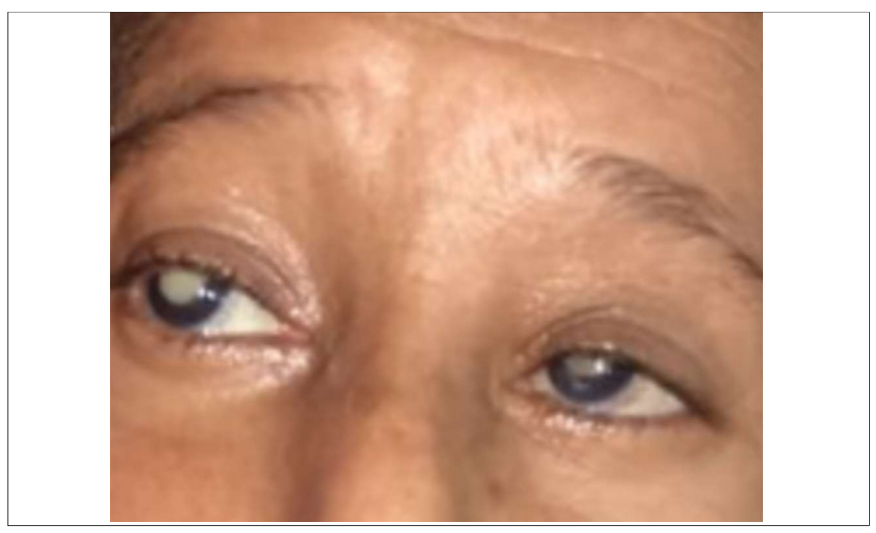

Figure 1: Bilateral blephroptosis with dense cataracts

years has been reported. ${ }^{4}$ Its prevalence is one in 4 - 8 million new births. These people biologically age faster than normal i.e., about ten years in a single year. It is interesting to note that such people have normal IQ and intelligence. ${ }^{5}$ Incidence of progeria is equal throughout the world. It has no gender, geographical or ethnic predisposition. There are about 114 diagnosed cases across 39 countries. ${ }^{5}$ The average age of survival is 13.5 years (with life expectancy about 8 - 21 years). "Mutations in the lamin A/C (LMNA) gene is responsible in majority of cases, but many other genetic pathways are also involved".2

In conclusion, our case is unique on account of old age of presentation and the development of cataracts in both eyes.

\section{CONFLICT OF INTEREST:}

Authors declared no conflict of interest.

\section{AUTHORS' CONTRIBUTION:}

MI: Involved in drafting the manuscript and revising it critically for important intellectual content.

$\mathrm{MI}, \mathrm{MH}$ : Contributed to conception and design of the work and intellectual properties related work; Made contributions to the analysis.

S: Contributed to the analysis.

\section{REFERENCES}

1. Ahmed MS, Ikram S, Bibi N, Mir A. Hutchinson-Gilford Progeria Syndrome: A premature aging disease. Mol Neurobiol 2017; 610-7.

2. Alves DB, Silva JM, Menezes TO. Clinical and radiographic features of Hutchinson-Gilford progeria syndrome: A case report. World J Clin Cases 2014; 2:67-71.

3. NHGRI Clinical Research on Progeria.available from: https:// www.genome.gov/11007255/learning-about-progeria/Last Updated: December 27, 2013.

4. Fukuchi K, Katsuya T, Sugimoto K, Kuremura M, Kim HD, Li L, et al. LMNA mutation in a 45-year-old Japanese subject with Hutchinson-Gilford progeria syndrome. J Med Genet 2004; 41:e67. 
5. Progeria Research Foundation (PRF) webpage. [accessed on May 17, 2014]. Available from:http://www.progeriaresearch.org/ meet_the_kids.html.

Mohammad Idris ${ }^{1}$, Mahfooz Hussain ${ }^{1}$ and Shafaatullah ${ }^{2}$

1 Department of Ophthalmology, Lady Reading Hospital, Medical Teaching Institute, Peshawar, Pakistan
2 Centre of Biotechnology and Microbiology, University of Peshawar, Peshawar, Pakistan

Correspondence to: Dr. Mohammad Idris, Department of Ophthalmology, Lady Reading Hospital, Medical Teaching Institute, Peshawar, Pakistan

E-mail: idrisdaud80@gmail.com

Received: January 01, 2019; Revised: May 14, 2019 Accepted: May 24, 2019

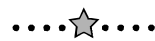

